\title{
Reduction of the Superconducting Gap of Ultrathin Pb Islands Grown on Si(111)
}

\author{
Christophe Brun, ${ }^{1}$ I-Po Hong, ${ }^{1}$ François Patthey, ${ }^{1}$ I. Yu. Sklyadneva,,${ }^{2,3}$ R. Heid, ${ }^{4}$ P. M. Echenique, ${ }^{2,5}$ K. P. Bohnen, ${ }^{4}$ \\ E. V. Chulkov, ${ }^{2,5}$ and Wolf-Dieter Schneider ${ }^{1}$ \\ ${ }^{1}$ École Polytechnique Fédérale de Lausanne (EPFL), Institut de Physique de la Matière Condensée, CH-1015 Lausanne, Switzerland \\ ${ }^{2}$ Donostia International Physics Center (DIPC), \\ Paseo de Manuel Lardizabal, 4, 20018 San Sebastián/Donostia, Basque Country, Spain \\ ${ }^{3}$ Institute of Strength Physics and Materials Science, Prospekt Academicheski 2/1, 634021, Tomsk, Russia \\ ${ }^{4}$ Forschungszentrum Karlsruhe, Institut für Festkörperphysik, P.O. Box 3640, D-76021, Karlsruhe, Germany \\ ${ }^{5}$ Departamento de Física de Materiales and Centro Mixto CSIC-UPV/EHU, Facultad de Ciencias Químicas, UPV/EHU, \\ Apartado 1072, 20080 San Sebastián/Donostia, Basque Country, Spain
}

(Received 12 December 2008; published 19 May 2009)

\begin{abstract}
The energy gap $\Delta$ of superconducting $\mathrm{Pb}$ islands grown on $\mathrm{Si}(111)$ was probed in situ between 5 and 60 monolayers by low-temperature scanning tunneling spectroscopy. $\Delta$ was found to decrease from its bulk value as a function of inverse island thickness. Corresponding $T_{c}$ values, estimated using bulk gapto- $T_{c}$ ratio, are in quantitative agreement with ex situ magnetic susceptibility measurements, however, in strong contrast to previous scanning probe results. Layer-dependent $a b$ initio density functional calculations for freestanding $\mathrm{Pb}$ films show that the electron-phonon coupling constant, determining $T_{c}$, decreases with diminishing film thickness.
\end{abstract}

DOI: 10.1103/PhysRevLett.102.207002

PACS numbers: 74.78.Na, 68.37.Ef, 74.50.+r, 74.78.Db

The fundamental question of how the superconducting properties of a material are modified when its thickness is reduced down to a few atomic monolayers has stimulated considerable theoretical and experimental interest since the 1960s [1-7]. Today, with the emergence of nanoscience and nanotechnology, this question is of special relevance for possible technological applications in superconducting nanodevices. The early model of Blatt and Thompson predicted an increase of the critical temperature $\left(T_{c}\right)$ above the bulk value with decreasing film thickness, together with $T_{c}$ oscillations due to quantum size effects [1]. However, if proper boundary conditions allowing for spillout of the electronic wave functions in thin films are taken into account, a decreasing $T_{c}$ with decreasing film thickness is predicted $[4,5,8-11]$. Depending on the material, early experimental results showed either a decrease $(\mathrm{Pb})$ or an increase ( $\mathrm{Al}, \mathrm{Ga}, \mathrm{Sn}, \mathrm{In})$ in $T_{c}$ on film thickness reduction $[12,13]$, mainly related to disorder in the films composed of small metallic grains [13]. In contrast, pioneering experiments on crystalline $\mathrm{Al}$ films reported no $T_{c}$ enhancement, allowing one to address intrinsic thickness-dependent properties of crystalline superconducting films [14].

Recently, $\mathrm{Pb}$ films grown on $\mathrm{Si}(111)$ attracted much attention in this context [15]. Ex situ resistivity [16] and magnetic susceptibility measurements [17] reported a decrease of $T_{c}$ with decreasing $\mathrm{Pb}$ film thickness. In contrast, recent in situ scanning tunneling spectroscopy (STS) investigations on $\mathrm{Pb} / \mathrm{Si}(111)$ islands reported no significant change in $T_{c}$ upon thickness reduction [18,19], while the very small $T_{c}$ oscillations observed were attributed to quantum size effects $[1,18]$. These contradictory experimental results call for a clarification.
In this Letter, we report in situ layer-dependent STS measurements of the energy gap of ultrahigh-vacuum grown single-crystal $\mathrm{Pb} / \mathrm{Si}(111)-(7 \times 7)$ and $\mathrm{Pb}-\sqrt{3} \times$ $\sqrt{3} / \mathrm{Si}(111)$ islands in the thickness range of 5-60 monolayers (ML). In contrast to previous STS studies on this system $[18,19]$, we show that the energy gap decreases with decreasing island thickness $d$ for both crystalline and disordered interfaces. Corresponding $T_{c}$ values, estimated using the bulk gap-to- $T_{c}$ ratio, decrease with a $-1 / d$ dependence, in quantitative agreement with ex situ measurements of Ozer et al. [17]. Moreover, employing layerdependent $a b$ initio density functional theory (DFT) calculations for freestanding $\mathrm{Pb}$ films, we find for thin layers a similar behavior of $T_{c}$, caused by a thickness-dependent decrease of the electron-phonon coupling.

$\mathrm{Pb}$ was thermally evaporated on the $\mathrm{Si}(111)-(7 \times 7)$ (hereafter $7 \times 7$ ) or on the $\mathrm{Pb}-\sqrt{3} \times \sqrt{3} / \mathrm{Si}(111)$ (in short $\mathrm{Pb} \sqrt{3}$ ) substrate [20] kept at room temperature favoring the growth of $\mathrm{Pb}$ single crystals with their (111) axis perpendicular to the surface $[15,20]$. The samples were studied at a temperature of $4.6 \mathrm{~K}$ or, by pumping on the He dewar, at $3.0 \mathrm{~K}$ in a homebuilt STM [21]. Cut PtIr wires were used as STM tips. Differential conductance $(d I / d V)$ measurements were performed with open feedback loop, using lock-in technique (modulation voltage of 0.2 to $0.5 \mathrm{mV}_{\mathrm{pp}}$ at $277 \mathrm{~Hz})$ at a typical tunneling resistance of $15 \mathrm{M} \Omega$. Radio frequency (rf) noise has been carefully filtered [2224].

Figure 1 shows a STM image of a flattop Pb island extending over two $\mathrm{Si}$ terraces separated by a single $\mathrm{Si}(111)$ step [25]. The island mainly consists of an $8 \mathrm{ML}$ thick $\mathrm{Pb}$ area with respect to the $\mathrm{Si}$ surface, as determined 


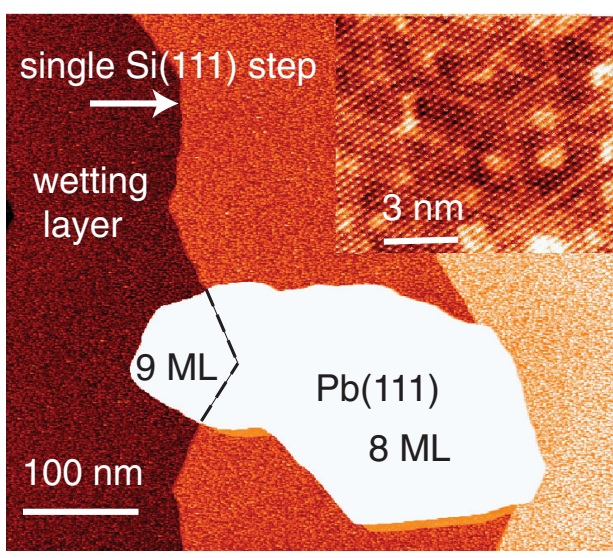

FIG. 1 (color). STM image of a flattop $\mathrm{Pb}(111)$ single-crystal island grown on $\mathrm{Si}(111)-(7 \times 7)$. The island extends over two $\mathrm{Si}$ terraces. Island thickness includes the wetting layer. $V_{\text {bias }}=$ $-1.0 \mathrm{~V}, I=100 \mathrm{pA}$. The inset shows a magnified view, revealing the $\mathrm{Pb}$ lattice with atomic resolution $\left(V_{\text {bias }}=20 \mathrm{mV}, I=\right.$ $1 \mathrm{nA})$.

from the apparent height in the STM topograph. The island thickness includes the $\simeq 1 \mathrm{ML}$ wetting layer [26]. The inset shows a magnified view of the $\mathrm{Pb}$ surface lattice with atomic resolution. The observed superstructure reflects the buried $7 \times 7$ interface [25]. $\mathrm{Pb}$ areas of constant thickness with a lateral extension larger than the $T=0 \mathrm{~K}$ $\mathrm{Pb}$ bulk coherence length $(\sim 80 \mathrm{~nm})$ were selected for this study, excluding transition regions where the island thickness changes.

Figure 2 displays a selected set of measured $d I / d V$ spectra for the indicated island thicknesses at $T=3.0 \mathrm{~K}$. Measurements have also been performed at $4.6 \mathrm{~K}$ (not shown). Each curve is an average of more than 10 individual $d I / d V$ spectra taken at various locations on one island. The background conductance was subtracted and the curves were normalized. The spectra display a clear superconducting energy gap $\Delta$ decreasing with decreasing island thickness. The observed small spectral asymmetry between positive and negative bias reflects the limits of the background subtraction on the $7 \times 7$ interface. Two phonon modes (indicated by arrows for the 60 ML curve) are clearly detected. Their energies (the difference between the local extrema in $d^{2} I / d V^{2}$ and $\Delta$ ) are $4.6 \pm 0.2$ and $8.5 \pm 0.2 \mathrm{meV}$, in excellent agreement with the values reported for bulk $\mathrm{Pb}$ [27].

In order to quantify the observed $\Delta$, a least squares analysis of the $d I / d V$ spectra is performed based on the standard expression for the tunneling conductance between a normal metal and a superconductor:

$$
\frac{d I}{d V}(V)=-G_{n n} \int_{-\infty}^{\infty} \frac{\rho_{s}(\epsilon)}{N(0)} f^{\prime}(\epsilon-e V) d \epsilon,
$$

where $f^{\prime}(\epsilon)$ is the derivative of the Fermi function, $G_{n n}$ is the Ohmic conductance of the junction, and

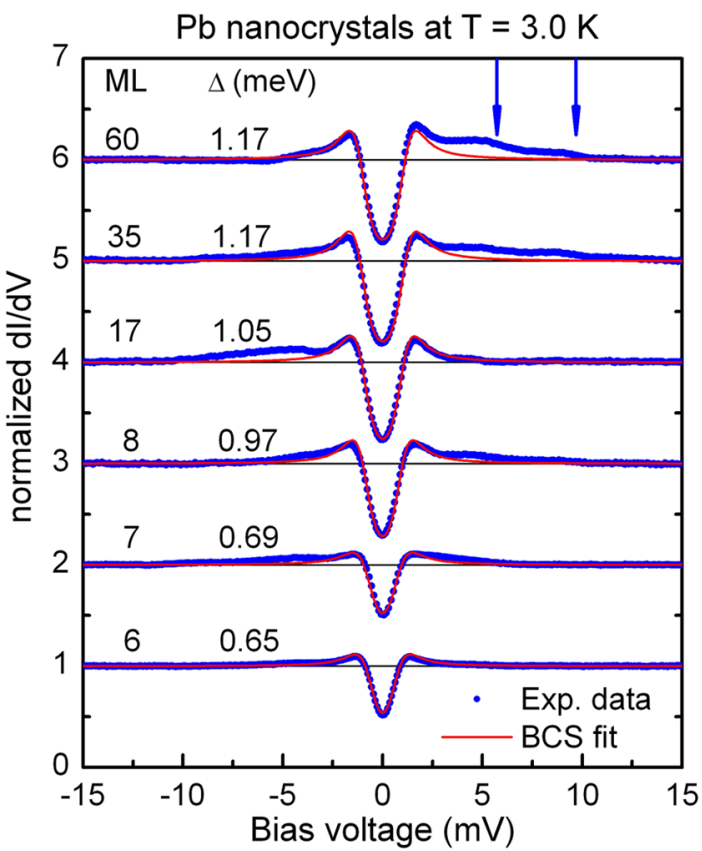

FIG. 2 (color online). Experimental (dots) and calculated (continuous lines) differential conductance spectra for tunneling between a PtIr tip and a large atomically flat $\mathrm{Pb}$ island of selected thickness. All spectra are measured at $3.0 \mathrm{~K}$ on $\mathrm{Pb} / \mathrm{Si}-(7 \times 7)$, except for the $7 \mathrm{ML}$ measured on $\mathrm{Pb} / \mathrm{Pb}-\sqrt{3} \times \sqrt{3} / \mathrm{Si}$. $\Delta_{\text {bulk }}(3.0 \mathrm{~K})=1.23 \mathrm{meV}$. Arrows indicate observed phonon modes. The zero conductance level is indicated for the $6 \mathrm{ML}$ spectrum. The other spectra are vertically displaced for clarity by integers.

$$
\frac{\rho_{s}(\epsilon)}{N(0)}=\operatorname{Re}\left\{\frac{|\epsilon|}{\sqrt{\epsilon^{2}-\Delta^{2}}}\right\}
$$

is the ratio between the superconducting and the normal density of states (DOS) of the $\mathrm{Pb}$ sample. The broadening introduced by the voltage modulation is taken into account by a proper convolution and the remaining rf noise voltage present in the tunnel junction by an additional convolution, assuming a Gaussian distribution [not written in Eq. (1)] $[22,23]$. The fitted standard deviation of the voltage noise is $280 \mu \mathrm{V} \mathrm{rms}$. The resulting $d I / d V$ spectra, shown in Fig. 2 (thin red lines), describe convincingly the experimental data. For $60 \mathrm{ML}, \Delta_{60 \mathrm{ML}}(3.0 \mathrm{~K})=1.17 \mathrm{meV}$, which is very close to the bulk value $\Delta_{\text {bulk }}(3.0 \mathrm{~K})=$ $1.23 \mathrm{meV}\left[\Delta_{\text {bulk }}(0 \mathrm{~K})=1.30 \mathrm{meV}\right]$. The observed relative reduction of the energy gap between 60 and $6 \mathrm{ML}$ at $3.0 \mathrm{~K}$ is $\approx 40 \%$.

The extracted gap values are averaged and plotted in Fig. 3(a) as a function of inverse film thickness. The energy gaps for both interfaces show a comparable reduction upon decreasing thickness. To allow for a comparison with previous results, the critical temperature $T_{c}$ was estimated from the measured gap values using the bulk $\Delta / T_{c}$ ratio (see theory below) and assuming the BCS temperature dependence of $\Delta(T)$. The results are displayed in Fig. 3(b). The continuous line represents a least squares analysis of the STS data, leading to the relation 


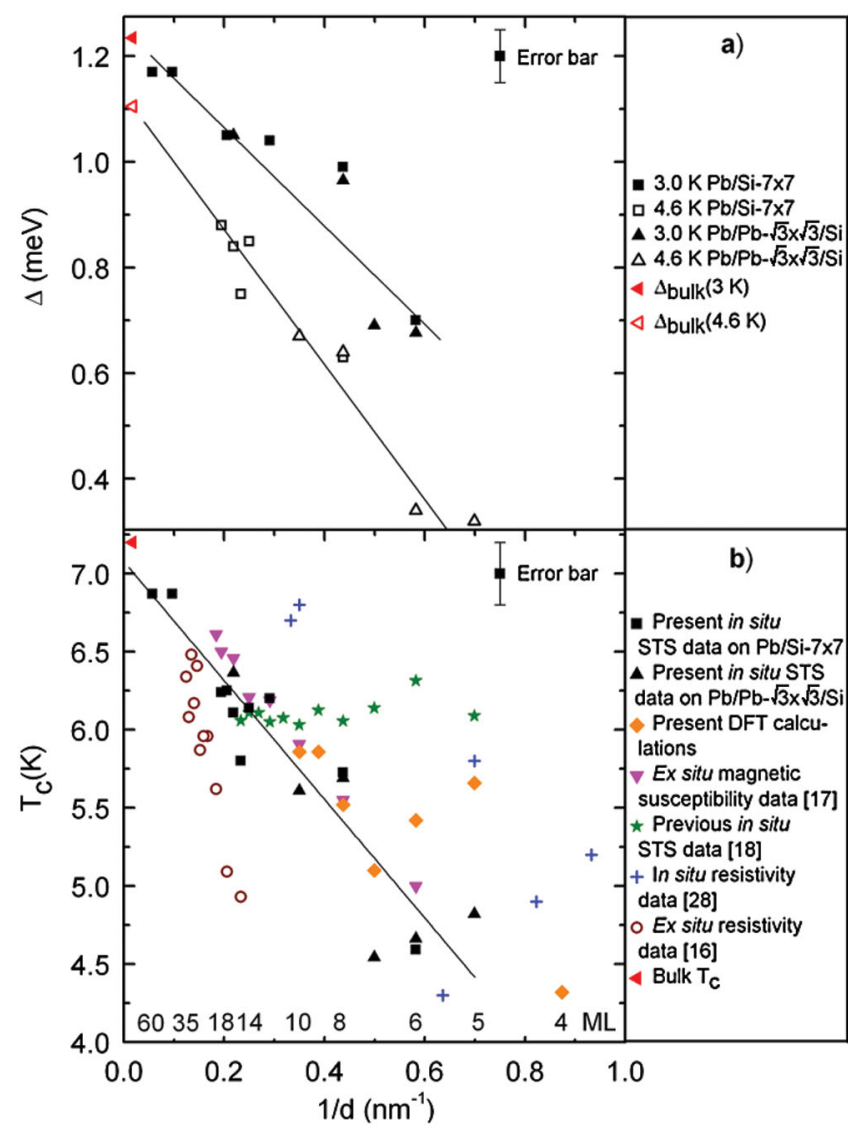

FIG. 3 (color). (a) Superconducting energy gap $\Delta$ as a function of inverse $\mathrm{Pb}$ island thickness $1 / d$, extracted from BCS fits of $d I / d V$ tunneling spectra, for the crystalline $(\mathrm{Pb} / \mathrm{Pb}-\sqrt{3} \times$ $\sqrt{3} / \mathrm{Si})$ and disordered $[\mathrm{Pb} / \mathrm{Si}-(7 \times 7)]$ interface. Continuous lines are guide for the eyes. (b) Estimated critical temperature $T_{c}$ as a function of $1 / d$, using the bulk $\Delta / T_{c}$ ratio and assuming BCS temperature dependence of $\Delta(T)$, to allow comparison with previously reported results. Continuous line is a fit to the present STS data. For both (a) and (b) error bars: Experimental dispersion and uncertainty in the fit results.

$T_{c}(d)=7.08 \times\left(1-d_{0} / d\right)$ with $d_{0}=1.88 \mathrm{ML}$. Consequently, for $d \leq 2$ ML the superconducting properties of $\mathrm{Pb}$ are expected to vanish.

Our estimated $T_{c}$ 's are in quantitative agreement with the ones of Ozer et al. [17], obtained ex situ on Ge capped $\mathrm{Pb} / \mathrm{Pb} \sqrt{3} \times \sqrt{3} / \mathrm{Si}(111)$. The trend observed in in situ resistivity measurements [28] is consistent with the present findings, the quantitative differences with respect to our data being most likely caused by the inhomogeneity of the films. Ex situ resistivity measurements [16] on Au capped $\mathrm{Pb} / \mathrm{Si}(111)-(7 \times 7)$ films show a much faster decrease of $T_{c}$ with decreasing film thickness than in our case, probably a consequence of the inverse proximity effect induced in the $\mathrm{Pb}$ film by the Au capping layer [29]. However, the previous in situ $\mathrm{STS}$ results on $\mathrm{Pb} / \mathrm{Si}(111)-(7 \times 7)$, which reported an essentially constant $T_{c}$ in this thickness range $[18,19]$, are at variance with our data. Furthermore, our observed energy gap reduction is much larger in amplitude than the very small oscillations of $T_{c}$ as a function of thickness [18], the amplitude of which is of only $2 \%$ of the average $T_{c}$.

In order to unravel the mechanisms responsible for the reduction of $T_{c}$ with decreasing $\mathrm{Pb}$ film thickness, we have performed ab initio calculations of the electronic structure (bands and density of states), phonon spectra, and electronphonon (e-ph) coupling properties. The gradient of the one-electron potential, the spectral Eliashberg function, and the mass-enhancement parameter $\lambda$ were computed for freestanding $\mathrm{Pb}(111)$ films in the range of 4-10 ML and for bulk lead [30]. We have also calculated the gap $\Delta$ and the critical temperature $T_{c}$ by solving the full nonlinear Eliashberg equations, and for comparison, the linearized gap equation of the Eliashberg theory [35,36]. In both calculations the obtained $T_{c}$ values agree within $\pm 0.02 \mathrm{~K}$ for each $\mathrm{Pb}$ film thickness considered. These calculations take full account of quantum size effects on the electron and phonon band structures and on the electron-phonon coupling properties. The evaluated ratios $2 \Delta(0 \mathrm{~K}) /\left(k_{B} T_{c}\right)$ are within $2 \%$ of the 4.19 bulk ratio, except for the $4 \mathrm{ML}$ film where the deviation is close to $5 \%$. This gives theoretical support for the assumption used to relate the measured $\Delta$ to $T_{c}$ values for each thickness.

The results are summarized in Fig. 4. Between 4 and $10 \mathrm{ML}$ the variations in $\lambda$ closely follow the variations in the electronic DOS at $E_{F}$ and the variations in the computed $T_{c}$ (see Fig. 3). For the $5 \mathrm{ML}$ film a quantum well state close to $E_{F}$ results in a peak in the DOS, which causes the peak in $\lambda$. A corresponding maximum is also found for $\Delta$ and $T_{c}$. The comparison of both the calculated DOS at $E_{F}$ and $\lambda$ for the $10 \mathrm{ML}$ film and the bulk suggests that, also for thicknesses larger than $10 \mathrm{ML}$, variations in the DOS at $E_{F}$ are the main reason for variations in $\lambda$ and $T_{c}$.

For all thicknesses studied theoretically, the largest contribution to the $e$-ph coupling originates from electronic states of $p_{z}$ symmetry: both surfacelike and bulklike $p_{z}$ states contribute to $\lambda$. The states of in-plane symmetry, $p_{x}$ and $p_{y}$, play a minor role in the $e$-ph coupling. The $e$-ph coupling matrix elements do not affect qualitatively the

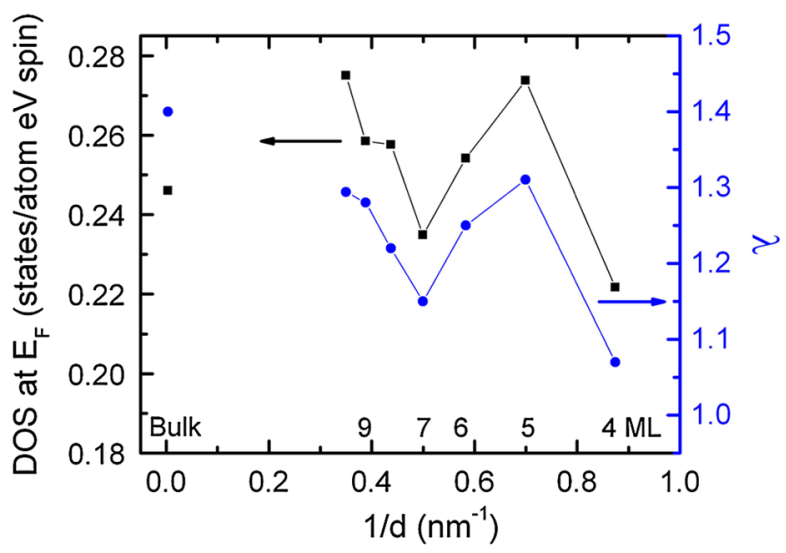

FIG. 4 (color online). Calculated electronic DOS at $E_{F}$ (squares) and electron-phonon coupling constant $\lambda$ (dots) for 4-10 ML freestanding $\mathrm{Pb}$ thin films and for bulk $\mathrm{Pb}$. 
phonon DOS $F(\omega)$ : the calculated Eliashberg function $\alpha^{2} F(\omega)$ shows the same peak structure as the phonon DOS $F(\omega)$, all phonon modes contributing to $\lambda$. This result is in good agreement with the absence of significant changes in the measured phonon energies in the $d I / d V$ spectra upon thickness reduction. Figure 3 shows that the theoretical $T_{c}$ 's are in fairly good agreement with the trend observed from the present STS data. The calculated $T_{c}$ 's for the 5, 6, and $7 \mathrm{ML}$ film are larger than those estimated from the measured gap values. This might be ascribed to the larger discrepancy arising at small film thickness between calculated and measured quantum-well states energies, the latter being smaller [26]. With decreasing film thickness the interaction with the substrate is expected to increasingly modify the electronic band structure and thus the DOS at $E_{F}, \lambda, \Delta$, and $T_{c}$, an effect not included in the calculations done for freestanding films.

For thin $\mathrm{Pb}$ islands on $\mathrm{Si}(111)$ the experimentally observed reduction of the superconducting energy gap with decreasing film thickness is consistent with the first principle results of a thickness-dependent $e$-ph coupling constant $\lambda$, where close to the ultrathin $\mathrm{Pb}$ film limit the variations of the density of states at $E_{F}$ play a decisive role. Interestingly, both atomically smooth $(\mathrm{Pb} / \mathrm{Pb}-\sqrt{3} \times$ $\sqrt{3} / \mathrm{Si})$ and disordered $[\mathrm{Pb} / \mathrm{Si}-(7 \times 7)]$ interfaces yield similar experimental behavior, in agreement with results showing that both systems are in the diffusive limit [17,37].

We acknowledge financial support of the Swiss National Science Foundation, the University of the Basque Country, the Departamento de Educación del Gobierno Vasco, and the Spanish Ministerio de Ciencia y Tecnología (MCyT) (Grant No. FIS 2004-06490-C03-01).

Note added in proof.-The recently reported pseudogaps mediated by quantum size effects in $\mathrm{Pb}$ islands [39] were also observed in our study and accounted for in the background correction of our conductance spectra.

[1] J. M. Blatt and C. J. Thompson, Phys. Rev. Lett. 10, 332 (1963).

[2] Yu. F. Komnik, E. I. Bukhshtab, and K. K. Man'kovskii, JETP 30, 807 (1970).

[3] M. Strongin, R. S. Thompson, O. F. Kammerer, and J. E. Crow, Phys. Rev. B 1, 1078 (1970).

[4] R. E. Allen, Phys. Rev. B 12, 3650 (1975).

[5] M. Yu, M. Strongin, and A. Paskin, Phys. Rev. B 14, 996 (1976).

[6] B. G. Orr, H. M. Jaeger, and A. M. Goldman, Phys. Rev. Lett. 53, 2046 (1984).

[7] D. B. Haviland, Y. Liu, and A. M. Goldman, Phys. Rev. Lett. 62, 2180 (1989).

[8] F. K. Schulte, Surf. Sci. 55, 427 (1976).

[9] J. Simonin, Phys. Rev. B 33, 7830 (1986).

[10] B. Chen, Z. Zhu, and X. C. Xie, Phys. Rev. B 74, 132504 (2006).

[11] A. A. Shanenko, M. D. Croitoru, and F. M. Peeters, Phys. Rev. B 75, 014519 (2007).
[12] B. Abeles, R. W. Cohen, and G. W. Cullen, Phys. Rev. Lett. 17, 632 (1966)

[13] A. M. Goldman and N. Markovic, Phys. Today 51, No. 11, 39 (1998).

[14] M. Strongin, O. F. Kammerer, H. H. Farrell, and D. L. Miller, Phys. Rev. Lett. 30, 129 (1973).

[15] M. Jalochowski and E. Bauer, Phys. Rev. B 38, 5272 (1988).

[16] Y. Guo et al., Science 306, 1915 (2004).

[17] M. M. Ozer, J. R. Thompson, and H. H. Weitering, Nature Phys. 2, 173 (2006).

[18] D. Eom, S. Qin, M.-Y. Chou, and C. K. Shih, Phys. Rev. Lett. 96, 027005 (2006).

[19] T. Nishio et al., Appl. Phys. Lett. 88, 113115 (2006).

[20] H. H. Weitering, D. R. Heslinga, and T. Hibma, Phys. Rev. B 45, 5991 (1992).

[21] R. Gaisch et al., Ultramicroscopy 42, 1621 (1992).

[22] H. Manoharan, C. Lutz, and D. Eigler (unpublished).

[23] H. Suderow et al., Phys. Rev. B 64, 020503 (2001).

[24] M. Ternes (private communication).

[25] I. B. Altfeder, K. A. Matveev, and D. M. Chen, Phys. Rev. Lett. 78, 2815 (1997).

[26] I.-P. Hong, C. Brun, F. Patthey, and W.-D. Schneider (to be published). If $1 \mathrm{ML}$ is added to the apparent thickness of the islands measured by STM, the energies of the quantum-well states measured by STS agree with the DFT calculated energies for freestanding Pb films [38].

[27] J. M. Rowell and L. Kopf, Phys. Rev. 137, A907 (1965).

[28] O. Pfennigstorf, A. Petkova, H.L. Guenter, and M. Henzler, Phys. Rev. B 65, 045412 (2002).

[29] P. G. De Gennes, Rev. Mod. Phys. 36, 225 (1964).

[30] We use density functional perturbation theory in the local density approximation and pseudopotential approach with a norm-conserving scalar-relativistic pseudopotential. [31-34]. We employed periodically repeated vertically relaxed films of $\mathrm{Pb}(111)$ separated by seven layers of vacuum. The lateral lattice constant was fixed to the theoretical bulk value of $a=4.87 \AA$. Valence states were expanded in plane waves up to a kinetic energy of 20 Ry. Surface Brillouin zone (SBZ) integrations were performed using 61 special points in the irreducible SBZ and the Gaussian smearing technique with a width of $0.2 \mathrm{eV}$. From the $a b$ initio $e$-ph coupling matrix elements we derived by Fermi-surface averaging the Eliashberg function $\alpha^{2} F$, from which the average $e$-ph coupling parameter $\lambda$ can be derived.

[31] R. Heid and K.-P. Bohnen, Phys. Rev. B 60, R3709 (1999).

[32] B. Meyer, C. Elsässer, and M. Fähnle, "FORTRAN90 Program for Mixed-Basis Pseudopotential Calculations for Crystals," Max-Planck-Institut für Metallforschung, Stuttgart (unpublished).

[33] D. Vanderbilt, Phys. Rev. B 32, 8412 (1985).

[34] L. Hedin and B. I. Lundqvist, J. Phys. C 4, 2064 (1971).

[35] G. Bergmann and D. Rainer, Z. Phys. 263, 59 (1973).

[36] The calculated $\alpha^{2} F$ and the same effective Coulomb interaction parameter $\mu^{*}=0.11$ for all slabs considered have been used.

[37] T. Nishio et al., Phys. Rev. Lett. 101, 167001 (2008).

[38] C. M. Wei and M. Y. Chou, Phys. Rev. B 66, 233408 (2002).

[39] K. Wang et al., Phys. Rev. Lett. 102, 076801 (2009). 